\title{
Genetic characterization and genome-wide association mapping for dwarf bunt resistance in bread wheat accessions from the USDA National Small Grains Collection
}

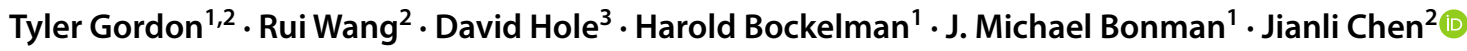

Received: 25 June 2019 / Accepted: 3 January 2020 / Published online: 14 January 2020

(c) The Author(s) 2020

\begin{abstract}
Key message Dwarf bunt-resistant bread wheat accessions and SNP markers associated with DB resistance identified in this study are valuable resources for characterization and deployment of DB resistance in bread wheat.

Abstract Dwarf bunt (DB), caused by Tilletia controversa J.G. Kühn, can significantly reduce grain yield and quality on autumn-sown wheat in regions with prolonged snow cover. DB can be managed with the use of resistant cultivars. The objectives of the present study were to characterize DB resistance in a large set of bread wheat accessions from the National Small Grains Collection and use a genome-wide association study approach to identify genetic loci associated with DB resistance. A total of 292 accessions were selected using historical DB resistance data recorded across many trials and years in the Germplasm Resources Information Network (GRIN) and re-tested for DB resistance in replicated field nurseries in Logan, UT, in 2017, 2018, and 2019. Ninety-eight accessions were resistant with DB normalized incidence $\leq 10 \%$, and twenty-eight of these were highly resistant with DB normalized incidence $\leq 1 \%$ in both GRIN and the field nurseries. Based on the presence of marker haplotypes of the four published dwarf bunt QTL on 6DS, 6DL, 7AL, and 7DS, highly resistant accessions identified in this study may provide novel resistance and should be further evaluated. This study validated one previously identified QTL on 6DS and identified an additional locus on 6DS. These loci explained 9-15\% of the observed phenotypic variation. The resistant accessions and molecular markers identified in the present study may provide valuable resources for characterization and deployment of DB resistance in bread wheat.
\end{abstract}

\section{Introduction}

Bread wheat (T. aestivum L.) is an important food staple and 772 million $\mathrm{t}$ were harvested globally in 2017 (FAOSTAT 2019). Dwarf bunt (DB), caused by the basidiomycete

Communicated by Albrecht E. Melchinger.

Electronic supplementary material The online version of this article (https://doi.org/10.1007/s00122-020-03532-0) contains supplementary material, which is available to authorized users.

Jianli Chen

jchen@uidaho.edu

1 USDA-ARS-Small Grains and Potato Germplasm Research Unit, 1691 S. 2700 W., Aberdeen, ID 83210, USA

2 University of Idaho-Aberdeen Research and Extension Center, 1693 S. 2700 W., Aberdeen, ID 83210, USA

3 Department of Plants, Soils and Climate, Utah State University, 2325 Old Main Hill, Logan, UT 84322, USA
Tilletia controversa J.G. Kühn [as 'contraversa'] in L. Rabenhorst (Kühn 1874), and common bunt (CB), caused by two closely related fungi Tilletia caries (DC.) Tul. \& C. Tul. [syn. T. tritici (Bjerk.) G. Wint.] and Tilletia laevis J. G. Kühn [syn. T. foetida (Wallr.) Liro], are destructive diseases of bread wheat and durum wheat (T. turgidum subsp. durum Desf.) (Goates 1996). While these three pathogens vary slightly in their spore morphology and etiology, they are closely related with similar modes of infection and means of control. DB and CB differ slightly; in that $T$. controversa infects autumn-sown wheat and requires several months of snow cover for teliospore germination on the soil surface, whereas $T$. caries and $T$. laevis primarily infect springplanted wheat from spores in the soil.

Initiation of DB and CB begins when dikaryotic infection hyphae penetrate emerging seedlings thereby infecting the developing apical meristem (Kollmorgen and Ballinger 1987). The resulting systemic infection is often cryptic until flowering, when the fungal hyphae invade and replace developing ovaries with darkly pigmented teliospores that 
comprise a fungal sorus or bunt ball (Goates 1996; Castlebury et al. 2005). Yield losses due to DB and CB can exceed $80 \%$, and trimethylamine emitted by the teliospores causes a fetid, rotting fish odor which reduces flour quality (Goates 1996; Castlebury et al. 2005).

Difenoconazole, a seed treatment fungicide, effectively controls both diseases without causing yield reductions or phytotoxicity (Keener et al. 1995; Goates 1996) though genetic resistance offers a cost-effective compliment to seed treatments particularly in organic production systems. Most of the wheat landraces in the USDA National Small Grains Collection (NSGC) were screened for bunt resistance over the past 30 years, and resistance was found primarily in germplasm originating from regions in Iran, Macedonia, Montenegro, Serbia, and Turkey (Bonman et al. 2006). However, resistance was relatively rare. Among 10,759 landrace accessions tested for CB resistance, only 597 (5.5\%) were resistant, and of 8,167 landrace accessions tested for DB resistance, only $104(1.3 \%)$ were resistant (Bonman et al. 2006).

DB and CB resistance is putatively controlled by genefor-gene interactions, and it is assumed that the same genes confer resistance to both diseases (Hoffman and Metzger 1976; Goates 2012). An expanded set of bunt differential wheat accessions representing $16 \mathrm{Bt}$ genes was developed to elucidate host-pathogen interactions (Goates 2012). Using these $B t$ differentials, Goates (2012) found 19 pathogenic races of $T$. controversa, 36 races of $T$. caries, and 15 races of T. laevis, and determined that Bt8 (PI 554120), Bt11 (PI 554119), and Bt12 (PI 119333) were broadly effective against most races of DB and CB.

Genomic tools in wheat including dense molecular marker arrays with annotations (Wang et al. 2014), genotyping by sequencing, and reference genome sequences (IWGSC 2018) have enabled the identification of genetic loci underpinning DB and CB resistance (Supplementary File 1). Linkage mapping (Chen et al. 2016; Singh et al. 2016; Steffan et al. 2017) and association mapping techniques (Bhatta et al. 2018; Mourad et al. 2018) have located bunt resistance loci on 19 wheat chromosomes. Identifying markers tightly linked to resistance will enable the discovery of additional resistance genes and introgression of multiple resistance genes into adapted cultivars.

The NSGC is a worldwide collection of the small grains and contains 42,544 bread wheat accessions. Of these, 19,378 accessions have been systematically characterized for DB resistance since the early 1980 s, and only $129(0.7 \%)$ are classified as resistant based on a DB incidence threshold of $\leq 10 \%$ proposed by Goates (2012). The purpose of this study was to: 1) verify the DB resistance in the NSGC bread wheat accessions with replicated field trials, and 2) identify genetic loci associated with DB resistance using a genomewide association study (GWAS) approach.

\section{Materials and methods}

\section{Plant materials}

DB resistant and susceptible accessions were selected for this panel based on data from the US National Germplasm System online database: Germplasm Resources Information Network (GRIN), accessed at https://npgsweb.arsgrin.gov/gringlobal/search.aspx. Using a resistance threshold of $\leq 10 \%$ disease incidence relative to the susceptible check (Goates 2012), only 129 GRIN accessions were classified as DB resistant. An additional seven accessions with DB incidence below $13 \%$ were also included in the panel for a total of 136 bread wheat accessions classified as resistant for the GWAS. In an attempt to mitigate the effects of population structure on the GWAS, one susceptible accession from the same geographic region as each resistant accession was selected. For example, PI 470452 was classified as resistant and originated in Agri Province, Turkey; therefore, a susceptible accession from Agri Province, Turkey, PI 470470, was also selected. Additionally, the bunt differentials (Goates 2012), including BtO through Bt15, Btp, and PI 173438 (unknown Bt), and two known susceptible winter cultivars 'Wanser' (CItr 13844) and 'Cheyenne' (CItr 8885), were also included in the GWAS panel. Supplementary File 2 lists the accession number, name, taxon, geographic origin, improvement status, pedigree, and DB incidence for each of the 292 accessions.

\section{Field trials}

Since the 1980s, GRIN DB normalized incidence (NI) relative to the susceptible check cultivar 'Cheyenne' in each trial was collected from NSGC accessions grown at the Green Canyon USDA-ARS disease screening nursery in Logan, UT (approximately $3 \mathrm{~km}$ east of Logan: $41^{\circ} 46^{\prime} 21.05^{\prime \prime} \mathrm{N}, 111^{\circ} 46^{\prime} 52.68^{\prime \prime} \mathrm{W}$, elevation $1450 \mathrm{~m}$ ). DB field trials conducted in 2017, 2018, and 2019 were evaluated near the Green Canyon site at the Utah State University (USU) Research Farm in Logan, UT (41 $45^{\circ} 46.46^{\prime \prime} \mathrm{N}$, $111^{\circ} 48^{\prime} 54.98^{\prime \prime} \mathrm{W}$, elevation $1400 \mathrm{~m}$ ). USU field trials were sown with a head row planter on October 10, 2016, September 27, 2017, and September 18, 2018 with one accession per 1-m row and two replications in 2017 and 2018 and one replication in 2019. Each row was inoculated after seedling emergence on November 4, 2016, October 24, 2017, and November 6, 2018, with approximately $100 \mathrm{ml}$ of a concentrated DB teliospore suspension $\left(2 \times 10^{6}\right.$ spores $\mathrm{ml}^{-1}$ water $)$. A composite of teliospores from infected spikes previously collected in the USU DB nursery were used for the inoculations. Disease incidence 
was assessed on fully mature adult plants, Zadoks stage 94 (Zadoks et al. 1974), on August 3, 2017, July 26, 2018, and August 6, 2019 by counting the number of spikes where at least one floret was infected, and dividing by the total number of spikes in the row. DB incidence per replicate was normalized to the average of the six plots of the susceptible cultivar, Wanser. Accession DB incidence and NI are reported in Supplementary File 2, and the mean DB NI for each field trial can be accessed through GRIN.

\section{Molecular marker assessment}

A modified DNA CTAB protocol was used to extract genomic DNA from seedlings at the 2-3 leaf stage (Babiker et al. 2015). A 2-cm segment of leaf tissue was placed into 96 well Corning ${ }^{\circledR}$ Costar $^{\circledR}$ tubes (Corning, NY, USA) and macerated in CTAB extraction buffer with a bead grinder. The aqueous layer was separated in chloroform, extracted, and the precipitate was washed with isopropanol and then ethanol. Resulting DNA pellets were suspended in Tris $(10 \mathrm{mM})$ and sent to the USDA-ARS Small Grains Genotyping Laboratory in Fargo, ND, where samples were genotyped using the $90 \mathrm{~K}$ iSelect SNP assay as described by the manufacturer (Illumina, San Diego, CA). Allele clustering was completed using Genome Studio v.2.0.2 (Illumina), and the resulting set of 41,511 polymorphic SNPs was exported to JMP Genomics v.9.0 (SAS Institute Inc., Cary, NC, USA) for filtering. Markers were excluded if minor allele frequency (MAF) was $<4 \%$, or missing data were $>10 \%$. Heterozygous calls were also removed. Accessions were classified as duplicates and removed if they were $\geq 99.7 \%$ identical across all polymorphic SNPs. A final group of 246 bread wheat accessions were selected, and 19,281 SNP markers were aligned with the physical wheat annotation (IWGSC 2018) and used for subsequent marker-trait associations.

\section{Statistical analyses}

Unless stated otherwise, all statistical analyses were conducted using JMP ${ }^{\circledR}$ Genomics v. 9.0. By design, the DB NI had a bimodal distribution, and a Shapiro-Wilk normality test (Shapiro and Wilk 1965) of trial residuals indicated a significant $(P<0.0001)$ shift from normality. Similarly, square root and $\log _{10}$ transformations of the trials indicated significant $(P<0.0001)$ deviations from normality, and the untransformed DB NI data were used in all further analyses. A mixed model with genotype set as a fixed effect and trial as a random effect was used to calculate best linear unbiased estimates (BLUEs) for DB NI across trials and replications (Henderson 1975). Broad-sense heritability $\left(\mathrm{H}^{2}\right)$ was calculated using the formula: $H^{2}=\sigma_{G}^{2} /\left[\sigma_{G}^{2}+\sigma_{E x G}^{2} / r+\sigma_{2}^{2} / r\right]$ where $\sigma_{G}^{2}$ is the genotypic variance, $\sigma_{E x G}^{2}$ is the interaction variance between trial and genotype, $\sigma_{e}^{2}$ is the residual variance, and $\mathrm{r}$ is the number of data sets (Hanson et al. 1956). Correlation coefficient estimates between trials were calculated using a Spearman's Rho nonparametric rank-sum correlation procedure.

Genome-wide linkage disequilibrium (LD) was calculated as $r^{2}$ values between each marker within chromosome groups (Supplementary File 3). An IBS familial relationship matrix ( $\mathrm{k}$ matrix) and heat map were generated using the Ward hierarchical clustering method (Ward Jr and Hook Ward and Hook 1963) to explore potential subpopulations within the panel. STRUCTURE v.2.3.4 (Pritchard et al. 2000) and STRUCTURE HARVESTER (Earl and vonHoldt 2012) software packages were used to optimize the number of subpopulations (k). In STRUCTURE, the burn-in iterations and Markov chain Monte Carlo replications were set to 10,000 , the admixture correlated model was selected, and five replicate iterations were performed. Proposed subpopulations with $\mathrm{k}$ between 1 and 10 were evaluated in STRU CTURE HARVESTER using the Evanno method (Evanno et al. 2005), and the number of subpopulations that corresponded with the highest $\Delta \mathrm{k}$ value was selected as the optimal model.

A principal component analysis with ten principal components (PCs) was generated (Q matrix) to explore population stratification, and the resulting scree plot was used to estimate the optimal number of PCs that would explain the most variation in the models (Price et al. 2006). Bayesian information content (BIC) assessments (Burnham and Anderson 2004) were used to formally test the various association analysis models. Tested models included a general linear model (GLM) without corrections for K or Q (the naïve model), a GLM that corrected for population stratification with 2, 3 or 5 PCs, and mixed linear models (MLMs) that controlled for both familial relationships, as a random effect, and population stratification with 2, 3 or 5 PCs as a fixed effect (Yu et al. 2006). All models correcting for familial relationships performed better than the Naïve model. A MLM with a kinship covariate matrix and two PCs had the lowest BIC value and was therefore chosen for further marker-trait association analysis.

Marker-trait associations between DB NI and SNP markers were conducted on trial means, and BLUEs. Resulting $P$ values were adjusted using an FDR multiple testing procedure (Benjamini and Hochberg 1995), and a significance threshold of $P \leq 0.05$ on FDR-adjusted $P$ values was used to identify SNP-trait associations for further analysis. SNPs significantly associated with DB NI in any trial or BLUE were aligned with the Chinese Spring reference genome sequence v1.0 (IWGSC 2018) using IWGSC BLAST (Alaux et al. 2018) with the highest coverage and identity location available. To assess potentially linked SNPs, the most significant marker in each putative marker-trait association group was included one at a time in the MLM as covariates. 
Markers in high LD with the covariate marker were no longer significantly associated with DB resistance and were grouped with the covariate SNP group.

\section{Results}

\section{Field trials}

Two susceptible check cultivars, Cheyenne and Wanser, showed a high incidence of DB in both USU field trials. The mean DB incidence for Wanser was $63.9 \%$ in $2017,82.8 \%$ in 2018 , and $67.3 \%$ in 2019, while Cheyenne had a mean DB incidence of $79.2 \%$ in $2017,84.6 \%$ in 2018 , and $88.6 \%$ in 2019. Across the three trials, all differentials showed consistent responses except for the $B t 9$ differential, which was classified as resistant in 2017 but susceptible in 2018 and 2019, and the Bt5 differential which was classified as susceptible in 2017 and 2019 but resistant in 2018 (Table 1).

A mixed model ANOVA (Supplementary File 3) found no significant trial effect, but there was a significant genotype and genotype-by-trial effect $(P<0.0001)$. Broadsense heritability $\left(\mathrm{H}^{2}\right)$ for DB NI was estimated at 0.93 . Best linear unbiased estimates derived from the mixed model of DB NI across trials are listed in Supplementary File 2. By design, the field trials were composed of approximately $50 \%$ resistant and $50 \%$ susceptible accessions as classified based on GRIN data (Fig. 1). USU field trials produced a similar response, with $50.3 \%$ showing resistance in $2017,50.7 \%$ showing resistance in 2018, and $45.2 \%$ with resistance in 2019 (Table 2). Accessions classified as susceptible based on GRIN showed a wide array of disease incidence in the field trials (Fig. 1). Most of the accessions classified as resistant based on GRIN data, yet susceptible in 2017, 2018, or 2019, were breeding lines from the USA. Another group of accessions, about half being landraces from Turkey, showed the opposite reaction; they were susceptible based on GRIN data, but resistant in the USU field trials (Supplementary File 2).

A Spearman's rank-sum nonparametric correlation was used to measure the degree of similarity between and among the GRIN, 2017, 2018, and 2019 USU field trial means and BLUEs. Correlation coefficients $\left(r^{2}\right)$ between data sets ranged from 0.70 and 0.93 , and all estimates were significant at $P<0.0001$. GRIN was correlated with the 2017, 2018, and 2019 trials, and BLUEs with correlation coefficients of $0.76,0.77,0.70$, and 0.85 , respectively. The correlation coefficient between the 2017, 2018, and 2019 USU trials was $0.88,0.76$, and 0.78 , respectively. In the 2017 USU field trials, the $r^{2}$ between replications was 0.86 , and in 2018 , the $r^{2}$ was 0.87 between the two replications.
Table 1 Bunt differential lines and known susceptible and resistant sources showing subpopulations, and dwarf bunt normalized incidence from the germplasm resources information network (GRIN), 2017, 2018, and 2019 Logan, UT field trials, and best linear unbiased estimates (BLUEs)

\begin{tabular}{|c|c|c|c|c|c|c|c|c|}
\hline Accession & Name & Bt gene & $\begin{array}{l}\text { Subpop- } \\
\text { ulation }\end{array}$ & GRIN & 2017 & 2018 & 2019 & BLUE \\
\hline CItr 8885 & Cheyenne & Susceptible & 4 & 100 & 119.1 & 102.2 & 131.6 & 116.2 \\
\hline CItr 13844 & Wanser & Susceptible & 4 & 100 & 100 & 100 & 100 & 104.4 \\
\hline PI 209794 & Heins VII & $\mathrm{BtO}$ & 1 & 97 & 135.3 & 85.2 & 130.2 & 111.2 \\
\hline PI 554101 & Selection 2092 & Btl & 1 & . & 103.3 & 92.1 & 101.7 & 104.0 \\
\hline PI 554097 & Selection 1102 & $B t 2$ & 1 & . & 121.6 & 98.1 & 131.4 & 119.2 \\
\hline CItr 6703 & Ridit & $B t 3$ & 3 & 76 & 10.5 & 42.6 & 67.2 & 51.2 \\
\hline PI 11610 & CI 1558 & Bt4 & 3 & 100 & 150.2 & 98.9 & 130.4 & 120.4 \\
\hline CItr 11458 & Hohenheimer & Bt5 & 1 & . & 69.4 & 3.4 & 26.7 & 32.1 \\
\hline CItr 10061 & Rio & Bt6 & 3 & . & 132.0 & 54.5 & 144.1 & 67.4 \\
\hline PI 554100 & Selection 50077 & $B t 7$ & 1 & 100 & 150.0 & 93.2 & 103.7 & 112.7 \\
\hline PI 554120 & M72-1250 & Bt8 & 2 & . & 0 & 3.0 & 1.9 & 7.5 \\
\hline PI 554099 & R63-6968 & $B t 9$ & 2 & . & 0 & 44.0 & 111.1 & 55.3 \\
\hline PI 554118 & R63-6982 & Bt10 & 2 & . & 17.9 & 19.0 & 69.3 & 37.0 \\
\hline PI 554119 & M82-2123 & Bt11 & 2 & 1 & 0 & 1.2 & 2.2 & 4.5 \\
\hline PI 119333 & 1696 & Bt12 & 6 & 0 & 0 & 0 & 0 & 3.4 \\
\hline PI 181463 & Thule III & Bt13 & 5 & 15 & 2.7 & 9.6 & 0.9 & 11.0 \\
\hline CItr 13711 & Doubbi & Bt14 & . & . & . & 0.0 & 2.8 & 3.7 \\
\hline CItr 12064 & Carleton & Bt15 & . & . & . & 9.6 & 15.5 & 14.8 \\
\hline PI 173437 & 7838 & $B t p$ & 6 & 0 & . & 0.7 & 0 & 0.1 \\
\hline PI 173438 & 7845 & Unknown & 6 & 0 & . & 0 & 0.9 & 0.1 \\
\hline PI 178383 & 6256 & $B t 8,9,10$ & 6 & 0 & 0 & 2.1 & 0 & 4.5 \\
\hline PI 476212 & SM Selection 4 & Unknown & 6 & 1 & 0 & 0 & 0 & 4.0 \\
\hline
\end{tabular}


Fig. 1 Dwarf bunt normalized incidence distributions across 292 wheat accessions from four data sets including the germplasm resources information network (GRIN), mean 2017, 2018, and 2019, Logan, UT field trials, and best linear unbiased estimates (BLUEs) from across trials; left pane: shaded accessions with DB normalized incidence $\leq 10 \%$ in GRIN, right pane: shaded accessions with DB normalized incidence $\geq 90 \%$ in GRIN
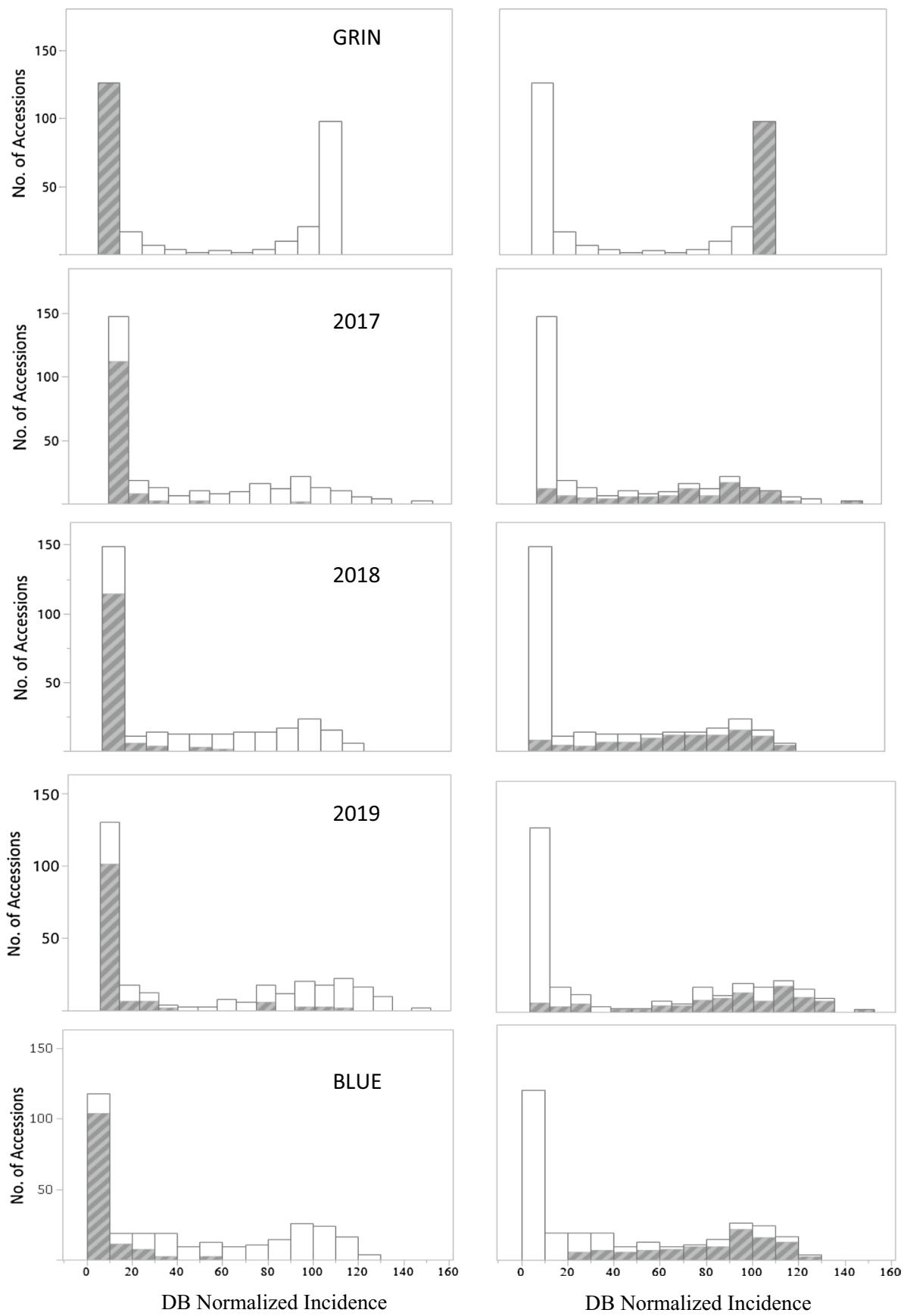

There were 98 accessions that were resistant with a DB NI $\leq 10 \%$ across GRIN and 2017, 2018, and 2019 USU trial means (Supplementary File 2). Of these, 28 were highly resistant with a DB NI $\leq 1 \%$ across all trials (Table 4). These highly resistant accessions included eight Turkish landraces, and 14 US lines with Turkish landraces in their pedigree. The remaining six highly resistant accessions were landraces from Serbia (1), Montenegro (1), Iran (1), and three breeding lines from the USA (Table 3 ).

\section{Population structure}

There were 44 accessions that were $\geq 99.7 \%$ identical across the 19,281 SNPs. These duplicate and near-duplicate accessions originated from similar geographic regions and had similar DB NI across data sets (Supplementary File 2), and were removed for further analyses. Genetic similarity among the 246 non-duplicated accessions ranged from 53\% to 99\% with a mean similarity of $67 \%$. 
Table 2 Number and percent of resistant and susceptible bread wheat accessions in four data sets and BLUEs and number of accessions that were consistent for resistance or susceptibility across all data sets

\begin{tabular}{llll}
\hline Data set $^{\mathrm{a}}$ & Resistant $^{\mathrm{b}}$ & Susceptible $^{\mathrm{c}}$ & $\begin{array}{l}\text { Percent } \\
\text { resistant } \\
(\%)\end{array}$ \\
\hline GRIN & 128 & 162 & 44.1 \\
2017 & 146 & 144 & 50.3 \\
2018 & 147 & 143 & 50.7 \\
2019 & 131 & 159 & 45.2 \\
BLUE & 116 & 174 & 40.0 \\
Consistent across & 98 & 116 & 45.8 \\
all trials & & & \\
\hline
\end{tabular}

${ }^{\text {a }}$ Data sets including the germplasm resources information network (GRIN), mean 2017, 2018, and 2019 Logan, UT field trials, and best linear unbiased estimate (BLUE) from across trails

${ }^{\mathrm{b}}$ Resistance classified as DB normalized incidence $\leq 10 \%$

${ }^{\mathrm{c}}$ Susceptibility classified as DB normalized incidence $>10 \%$

Table 3 Geographic origin and number of bread wheat accessions highly resistant, resistant, and susceptible to dwarf bunt (DB) across all data sets with the number of landraces within each group shown in parenthesis

\begin{tabular}{llll}
\hline Accession Origin & \multicolumn{3}{l}{$\mathrm{DB}$ resistance category } \\
\cline { 2 - 4 } Country & $\mathrm{HR}^{\mathrm{a}}$ & $\mathrm{R}^{\mathrm{b}}$ & $\mathrm{S}^{\mathrm{c}}$ \\
\hline Azerbaijan & 0 & 0 & $3(2)$ \\
Germany & 0 & 0 & 1 \\
Iran & $1(1)$ & $8(8)$ & $10(10)$ \\
Montenegro & $1(1)$ & $1(1)$ & $2(2)$ \\
Russia & 0 & 1 & $1(1)$ \\
Serbia & $1(1)$ & $6(6)$ & $9(9)$ \\
Spain & 0 & 0 & $1(1)$ \\
Turkey & $8(8)$ & $26(25)$ & $17(13)$ \\
USA & 17 & 56 & 72 \\
Total & 28 & 98 & 116 \\
\hline
\end{tabular}

${ }^{\text {a}}$ Highly resistant accessions with a DB NI $\leq 1 \%$

${ }^{\mathrm{b}}$ Resistant accessions with a DB NI $\leq 10 \%$

${ }^{\mathrm{c}}$ Susceptible accessions with a DB NI $>10 \%$

Based on the STRUCTURE HARVESTER $\Delta \mathrm{k}$ values, there were six distinct subpopulations $(\mathrm{k}=6)$ in the panel, and these groupings were supported by visual assessment of the Ward hierarchical clustering heat map and principal component analysis (Fig. 2a, b). Subpopulations based on the marker data corresponded well with geographic origin (Table 4). Subpopulation 1 and 4 consisted primarily of breeding lines and cultivars from the USA. Subpopulation 2 consisted of accessions from Turkey and breeding lines from the USA. Serbian landraces predominated in subpopulation 3, while landraces from Iran were primarily located
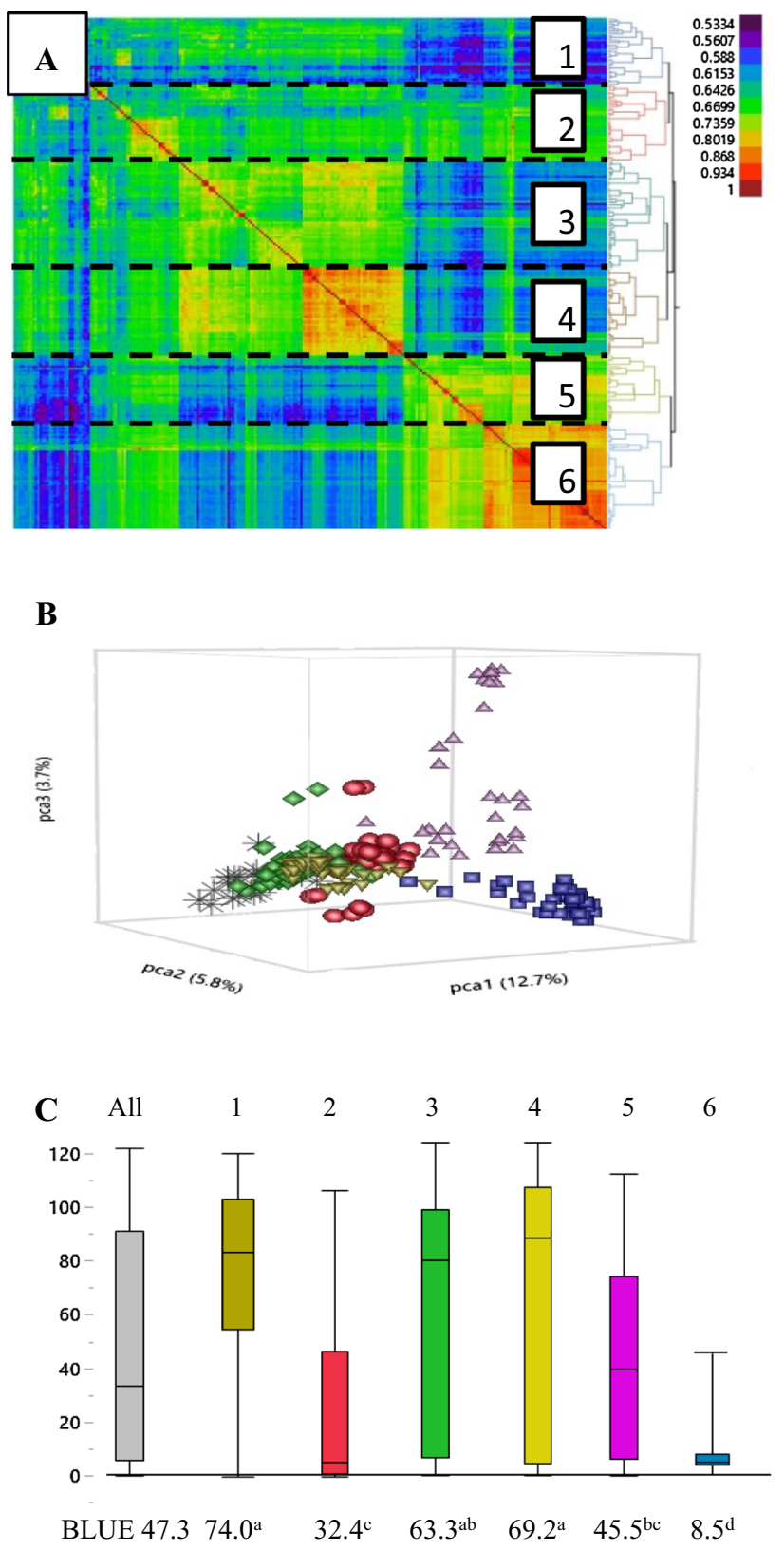

Fig. 2 a Genetic similarity heat map derived from an identity-by-state relationship matrix of 246 by 246 bread wheat accessions, regions of high (red) and low (purple) similarity between accessions; and a dendrogram showing six subpopulations (1-6) each separated by a dashed line. b Accessions plotted with three principal components showing subpopulations: 1 (brown stars), 2 (red circles), 3 (green diamonds), 4 (brown triangles), 5 (purple triangles), 6 (blue squares). c Best linear unbiased estimate (BLUE) dwarf bunt normalized incidence quantile box plots, left to right: all 246 accessions (gray) and subpopulations: 1 (blue), 2 (red), 3 (green), 4 (yellow), 5 (purple), 6 (blue); mean BLUE values are listed for each subpopulation below their respective box plots, means followed by a common letter are not significantly different by Tukey's HSD at $P \leq 0.05$ (color figure online) 
Table 4 Geographic origin and number of bread wheat accessions in each subpopulation

\begin{tabular}{lcccccc}
\hline Accession origin & \multicolumn{2}{l}{ Subpopulation } & & & \\
\cline { 2 - 6 } Country & 1 & 2 & 3 & 4 & 5 & 6 \\
\hline Azerbaijan & 0 & 0 & 1 & 0 & 2 & 0 \\
Germany & 1 & 0 & 0 & 0 & 0 & 0 \\
Iran & 0 & 5 & 0 & 0 & 19 & 3 \\
Montenegro & 1 & 0 & 2 & 1 & 0 & 0 \\
Russia & 0 & 0 & 1 & 0 & 1 & 0 \\
Serbia & 2 & 1 & 15 & 0 & 0 & 0 \\
Spain & 1 & 0 & 0 & 0 & 0 & 0 \\
Sweden & 0 & 0 & 0 & 0 & 1 & 0 \\
Turkey & 4 & 25 & 1 & 0 & 11 & 42 \\
USA & 24 & 16 & 31 & 43 & 0 & 36 \\
Total & 33 & 47 & 51 & 44 & 34 & 81 \\
\hline
\end{tabular}

in subpopulation 5. Landraces from Hakkari province, Turkey, and breeding lines from the USA that had Turkish landraces in their pedigree, were grouped into subpopulation 6 (Table 4). The bunt differentials were distributed across all the subpopulations (Table 1).

The BLUE DB NI estimate for the entire panel was $47.3 \%$ (Fig. 2c), and BLUE values for each subpopulation differed significantly at $P<0.0001$. Subpopulation 6 had the lowest mean BLUE DB NI of $8.5 \%$, and subpopulation 1 had the highest mean BLUE DB NI of $74.0 \%$. Of the 98 accessions that were resistant across trials (Table 3), $7 \%$ were in subpopulation $6,13 \%$ were in subpopulation 2 , with the remainder in subpopulations $1,3,4$, and 5 . Of the 28 highly resistant accessions, $75 \%$ were in subpopulation $6,7 \%$ were in subpopulation 2 and 3, and $4 \%$ were in each of the subpopulations 1,4 , and 5 .

\section{Linkage disequilibrium}

Genome-wide marker-pair $r^{2}$ correlations between 19,281 SNPs were plotted as a function of intrachromosomal intermarker genetic distance (Supplementary File 4). A median $r^{2}$ of 1 was found between SNP markers that were completely linked with an inter-marker physical distance of $0 \mathrm{Mbp}$. LD median $r^{2}$ decreased to 0.1 at an inter-marker distance of 0.1 to $1 \mathrm{Mbp}$ indicating an LD decay rate of $90 \%$ over the $1 \mathrm{Mbp}$ interval.

A smoothing spline curve with lambda equal to 10,000 was fit to the LD scatter plot to determine a genome-wide QTL confidence interval (Supplementary File 4). Others (Maccaferri et al. 2015; Liu et al. 2017) have used an LD of $r^{2}=0.3$ as a threshold for genome-wide QTL confidence intervals in wheat. In the present study, the largest spline curve $r^{2}$ value was 0.45 . When the smoothing spline curve was set to $r^{2}=0.3$, the physical distance was $0.67 \mathrm{Mbp}$, and when the curve was set to $r^{2}=0.1$, the distance was 6.80 Mbp.

\section{Marker-trait associations}

After controlling for kinship and population stratification, GWAS revealed four SNPs significantly (FDR-adjusted $P<0.05)$ associated with DB incidence in at least one trial or BLUE (Table 5, Fig. 3, and Supplementary Files 5, 6, 7 and 8). FDR-adjusted negative $\log _{10} P$ values for BLUE DB NI from these six marker-trait association groups ranged from 1.7 to 5.1, phenotypic variance $\left(r^{2}\right)$ ranged from 0.09 to 0.15 , and average DB NI BLUE values for accessions carrying resistance alleles ranged from 16.1 to 40.8 (Table 5). One marker-trait association group represented by two SNPs on chromosome 6DS was significant in three of the data sets (Table 5). Marker-trait association groups aligned with the 246 bread wheat accessions used for the GWAS (Supplementary File 9) show a corresponding decrease in DB NI as the number of resistant allele haplotypes increases (Supplementary File 10).

\section{Discussion}

Uniform DB infection requires specific environmental conditions that include several weeks of stable cool soil temperatures, a moist environment at the soil surface, and low light levels. These conditions are most reliably provided by continuous snow cover and are critical for teliospore germination (Chen et al. 2016). The two susceptible check cultivars, Wanser and Cheyenne, showed high DB incidence in all field trials indicating that the environmental conditions favored infection by the DB pathogen.

There were 28 highly resistant accessions with a DB $\mathrm{NI} \leq 1 \%$ across all data sets. Twenty-one of these highly resistant accessions either originated in Turkey or have Turkish landraces in their pedigrees. Similarly, the four bunt differentials that were highly resistant across trials, PI 554119 (Bt11), PI 119333 (Bt12), PI 173437 (Btp), and 
PI 173438 (unknown Bt), all either originated in Turkey or had a Turkish landrace in their pedigree. PI 119333 and PI 173437 had similar haplotype profiles (Table 6), and they shared SNP marker haplotypes with some of the other highly resistant accessions (Supplementary File 9). For instance, PI 119333 (Bt12) shares a similar haplotype profile to six other highly resistant accessions, and PI 173438 (with unknown $B t$ ) shares a similar profile with two other highly resistant accessions including PI 476212 (Table 6).

Based on the pedigree analysis (Supplementary File 2), many highly resistant breeding lines are derived from resistant Turkish landraces. PI 178383 and PI 476212 are in the pedigrees of several DB resistant cultivars, such as 'Weston,' 'DW,' 'Golden Spike,' and 'UI Silver' (Hole et al. 2002). However, some highly resistant landraces PI 345106 from Serbia, PI 345428 from Montenegro, and PI 627677 from Gilan province, Iran, have unique haplotypes and geographic origins (Table 6). Therefore, mapping the DB resistance within these unexploited resistance sources is an important step toward future molecular breeding for DB resistance.

In the present study, accessions were selected based on a DB NI resistance threshold of $\leq 10 \%$. Other accessions with intermediate levels of resistance are of interest to geneticists and plant breeders as they may contain a complex of minor or partial resistance genes. Specifically, PI 362710 from Montenegro, PI 345480 from Serbia, and PI 636153 a breeding line from Idaho, USA, had intermediate levels of DB resistance across data sets. Additionally, in the GRIN database, there are 976 bread wheat accessions that have a DB incidence recorded between 11 and 30\%. Environmental conditions can make bunt disease incidence variable from one year to the next. Thus, to confirm the partial resistance that may exist in these accessions, more research is warranted. Single-seed derived lines of each accession could be tested for multiple years in the field. Alternatively, molecular marker-assisted evaluation could be undertaken to identify accessions that do not carry known resistance QTL haplotypes. A quantitative PCR assay, like those developed for rust diseases (Admassu-Yimer et al. 2019), that reliably measures the degree of tissue colonization by the bunt pathogen could also provide a means for assessing partial resistance to the disease under greenhouse conditions.

Six subpopulations were selected in this panel of 246 bread wheat accessions based on $\Delta \mathrm{k}$ value optimization using STRUCTURE and STRUCTURE HARVESTER. These six subpopulations roughly corresponded to the geographic origins listed in GRIN (Table 5). We attempted to control for population relatedness by selecting both resistant and susceptible accessions from the same geographic area. Unfortunately, the subpopulations differed significantly in their levels of DB incidence (Fig. 2c) which could affect marker-trait associations. Specifically, those accessions in subpopulation 6 which corresponded with a Hakkari 


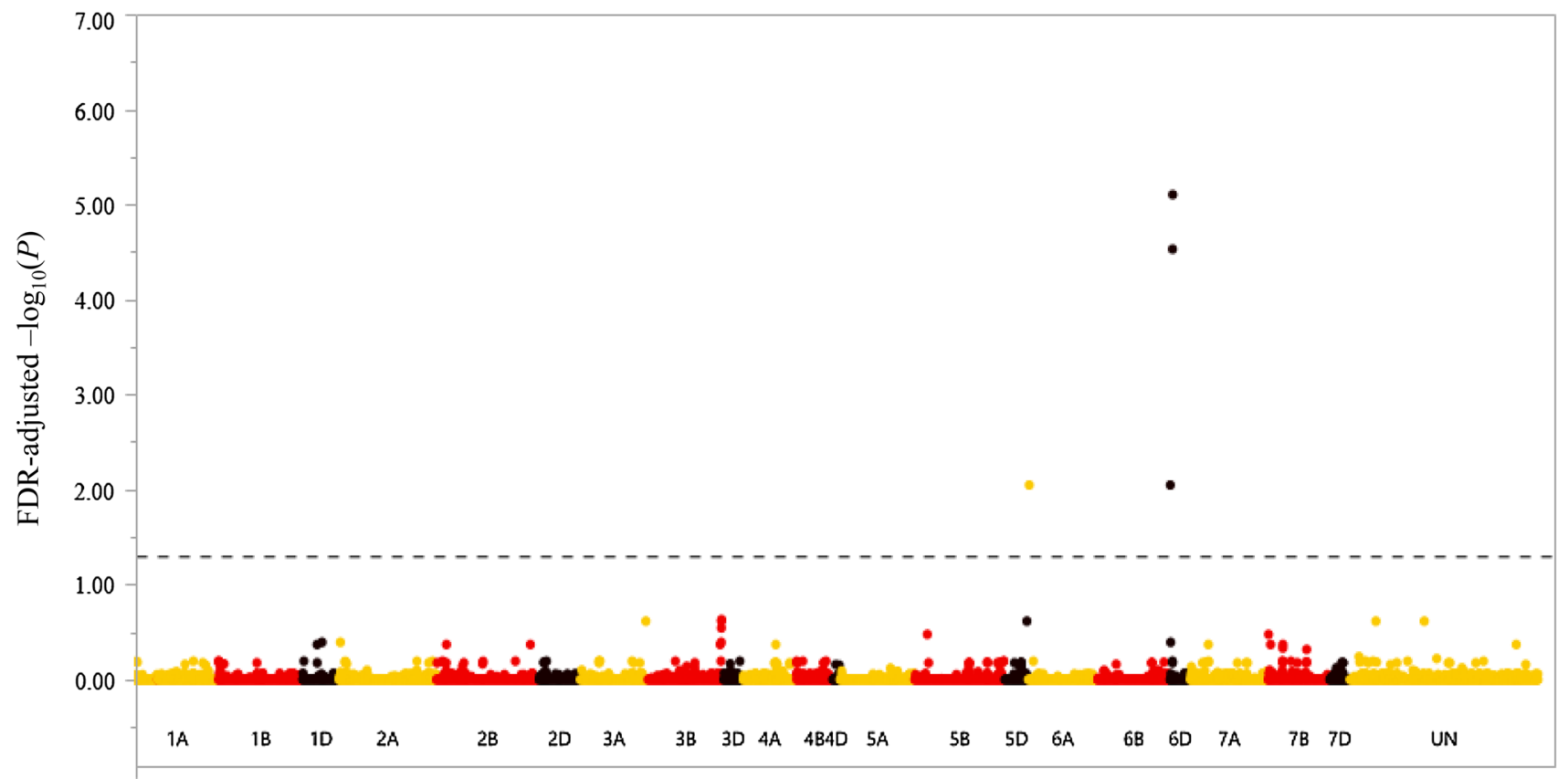

Fig. 3 Manhattan plot showing associations between 19,281 SNP markers and dwarf bunt normalized incidence best linear unbiased estimates (BLUEs) across 246 bread wheat accessions; the horizon- tal dashed line indicates an FDR-adjusted significance threshold of $P=0.05$; A-, B- and D-genome SNP markers are represented by yellow, red, and black dots, respectively (color figure online) province, Turkey origin, had significantly lower DB NI values than the other five subpopulations. Investigators may need to limit the origin of accessions to one region or locality to better balance population structure when designing future bunt GWAS. For instance, it might be of interest to examine all landrace accessions from Turkey as one study, and all landrace accessions from Iran as a separate study.

Broad-sense heritability, 0.93, was high for DB NI in this panel. Others have also reported high broad-sense heritability estimates for bread wheat resistance to dwarf bunt, 0.88-0.93, (Chen et al. 2016) and common bunt, 0.58-0.78 (Bhatta et al. 2018; Mourad et al. 2018). Although the broadsense heritability estimate and correlations between replications and years were high in this study, there were no significant SNPs that were consistent between data sets and met the FDR-adjusted $P$ value threshold of 0.05 (Supplementary File 8). Less stringent significance thresholds have been used in other bread wheat GWAS panels with small population sizes (Zegeye et al. 2014; Gao et al. 2016). A less stringent threshold would allow identification of additional markertrait associations in this panel (Supplementary File 7), but would increase the likelihood of false positive associations.

Of the two marker-trait associations that were significant in the present study (Table 5), only one corresponds with a previously reported QTL for DB or CB resistance (Supplementary File 1). Menzies et al. (2006) and Singh et al. (2016) found a QTL on 6DS with a peak marker at 6.17 $\mathrm{Mbp}$, which is likely the same QTL identified as $D B-6 D 2$ in this study. $D B-6 D 2$ is composed of two SNP markers and is most significantly associated with resistance identified in the present study (Table 5). Accessions containing the resistance alleles had a mean DB NI BLUE value of 16.3 (Supplementary File8). The Bt10 differential PI 178383 and another 30 accessions in this GWAS panel have this resistance-associated haplotype (Supplementary File 9). Based on the physical position, this QTL spanned a relatively narrow section of the chromosome from 6.97 to $7.29 \mathrm{Mbp}$, which is within the flanking position of the $\mathrm{Bt} 10$ gene (Menzies et al. (2006). Markers in this region can be developed and used in marker-assisted selection, but must first be validated in biparental populations.

Additionally, Menzies et al. (2006) hypothesized that the Bt10 QTL contributed by the bread wheat cultivar 'AC Cadillac' was closely linked with effective Ug99 stem rust resistance genes on 6DS, SrTmp or SrCad (Hiebert et al. 2016; Kassa et al. 2016). To determine whether Bt10 confers a stem rust resistance phenotype like $\operatorname{SrCad}$ or $\operatorname{SrTmp}$, PI 554118 (Bt10) and PI 178383 were screened with several Ug99 stem rust races. These two accessions were resistant to many of the same stem rust races as lines containing SrTmp and $\mathrm{SrCad}$ (unpublished data). Further studies are needed to determine if the 6DS region contains one or more genes that confer resistance to $\mathrm{DB}, \mathrm{CB}$, and wheat stem rust.

Two major QTL, Q.DB.ui-7DS (Chen et al. 2016), Q.DB-6DL (Steffan et al. 2017; Wang et al. 2019) and Q.DB.ui-7AL (Wang et al. 2019) that were previously 
Table 6 Highly resistant landrace accessions with dwarf bunt (DB) normalized incidence $\leq 1 \%$ across all data sets, bunt differentials and several known resistant and susceptible accessions with corre- sponding best linear unbiased estimates (BLUE) values and the presence $(+)$ or absence $(-)$ of the resistant allele haplotypes from each marker-trait association group detected in this study

\begin{tabular}{|c|c|c|c|c|c|c|c|c|}
\hline Accession & Bt gene & Origin & BLUE & $D B-6 D 1$ & $D B-6 D 2$ & $Q D B \cdot u i-6 D L^{a}$ & $Q D B \cdot u i-7 A L^{b}$ & $Q D B \cdot u i-7 D S^{c}$ \\
\hline PI 345106 & & Serbia & 3.9 & + & - & - & - & - \\
\hline PI 345428 & & Montenegro & 4 & - & - & - & - & - \\
\hline PI 476212 & & USA & 4 & + & - & - & - & + \\
\hline PI 560601 & & Turkey & 3.8 & + & - & - & - & - \\
\hline PI 560602 & & Turkey & 3.8 & + & - & - & - & - \\
\hline PI 560842 & & Turkey & 3.8 & + & - & - & - & + \\
\hline PI 560843 & & Turkey & 3.8 & + & - & - & - & - \\
\hline PI 560848 & & Turkey & 3.8 & + & - & - & - & + \\
\hline PI 627677 & & Iran & 4.1 & + & - & - & - & - \\
\hline CItr 8885 & Susceptible & USA & 116.2 & - & - & - & - & - \\
\hline PI 209794 & Susceptible & Germany & 111.2 & + & - & - & - & - \\
\hline PI 554101 & Btl & USA & 104 & - & - & - & - & - \\
\hline PI 554097 & $B t 2$ & USA & 119.2 & - & - & - & - & - \\
\hline CItr 6703 & Bt3 & USA & 51.2 & + & - & - & - & - \\
\hline PI 11610 & Bt4 & USA & 120.4 & + & - & - & - & - \\
\hline CItr 11458 & Bt5 & USA & 32.1 & + & - & - & - & - \\
\hline CItr 10061 & Bt6 & USA & 67.4 & + & + & - & - & - \\
\hline PI 554100 & $B t 7$ & USA & 112.7 & - & - & - & - & - \\
\hline PI 554120 & Bt8 & USA & 7.5 & + & - & - & - & - \\
\hline PI 554099 & $B t 9$ & USA & 55.3 & - & - & + & - & - \\
\hline PI 554118 & Bt10 & USA & 37 & + & + & - & - & - \\
\hline PI 554119 & Bt11 & USA & 4.5 & + & - & - & - & - \\
\hline PI 119333 & Bt12 & Turkey & 3.4 & + & - & - & - & + \\
\hline PI 181463 & Bt13 & Sweden & 11 & + & - & - & - & - \\
\hline PI 173437 & Btp & Turkey & 0.1 & + & - & - & - & - \\
\hline PI 173438 & $B t$ (unknown) & Turkey & 0.1 & + & - & - & - & + \\
\hline PI 178383 & Bt8,9,10 & Turkey & 4.5 & + & + & + & - & - \\
\hline
\end{tabular}

${ }^{a}$ The 6DL haplotype SNP markers are reported in Wang et al. (2019)

${ }^{b} 7 A L$ haplotype SNP markers are reported in Wang et al. (2019)

c7DS haplotype SSR markers are reported in Chen et al. (2016), and SNP markers were reported by Rui Wang (personal communication)

reported in biparental populations, were not detected in this study. The QTL Q.DB.ui-7DS was reported in the 'Rio Blanco'/'IDO444' population on 7DS with a peak marker, $w P t-2565$, at $5.9 \mathrm{Mbp}$ near the telomere (Chen et al. 2016). Based on pedigree information, the resistance in IDO444 was thought to be derived from PI 476212, the same parent contributing resistance in cv. 'Blizzard.' PI 476212 was initially selected for snow mold and DB resistance (Sunderman et al. 1986) and is in the pedigree of resistant cultivars 'DW' (PI 620629), 'Bonneville' (PI 557015), 'Golden Spike' (PI 614813), and 'UI Silver' (PI 658467). PI 476212 was highly resistant in the present study and was $99.99 \%$ similar to PI 173438 (unknown Bt) across the 19,281 SNPs, but the 7DS QTL reported by Chen et al. (2016) was not detected, possibly because too few accessions with this QTL were included in the present study. A haplotype analysis using SNPs in the 7DS region indicated that three of the highly resistant landraces in addition to the Bt12 differential and PI 476212 may contain the 7DS QTL (Table 6, Supplementary File 9).

Similarly, $B t 9$ has been mapped to 6DL between 172.8 and $175.9 \mathrm{Mbp}$ in a population derived from the $B t 9$ differential PI 554099 (Steffan et al. 2017). However, our GWAS did not detect any markers significantly associated with $6 \mathrm{DL}$ in any of the data sets. Using a biparental mapping population derived from a University of Idaho wheat breeding line 'IDO835', Wang et al. (2019) found two QTL for DB resistance, one on 6DL corresponding with the $B t 9$ locus, and one on $7 \mathrm{AL}$. We used the resistant haplotypes for both loci to find accessions that contain these QTL (Table 6, Supplementary File 9). The Bt9 differential and PI 178383 contained the haplotype profile for 
the 6DL locus, but none of the highly resistant accessions contained the 6DL or 7AL haplotype (Table 6, Supplementary File 9).

Aside from the possible low frequency of certain known loci in our GWAS panel, SNP maker filtering could also have reduced detection of known loci. SNP markers were filtered at a MAF threshold of $4 \%$ and any marker with fewer than thirteen individuals in each allelic state would have been filtered before analysis. This filtering threshold could mask SNP-trait associations that were present at low frequencies. To find such QTL, biparental populations could be developed with resistant accessions from the panel that lack alleles for the previously identified QTL.

Several marker-trait groups were associated with specific subpopulations (Supplementary File 11). For instance, $48 \%$ of accessions with the $Q D B$ - $6 D 2$-resistant haplotype are in subpopulation 4 (Supplementary Files 9 and 11). All the highly resistant accessions and 12 of the bunt differentials contained the $D B-6 D 1$ haplotype group. Conversely, the resistant haplotype for $D B-6 D 2$ was strongly associated resistance (Table 5); however, none of the highly resistant landrace accessions contained this haplotype (Table 6, Supplementary File 9).

The present study evaluated the DB responses recorded in the GRIN database for 292 wheat accessions rated in three field trials and identified 98 accessions that were resistant and 28 accessions that were highly resistant across all three years of USU field trials and in GRIN. Additionally, four SNP markers associated with DB resistance were identified, one marker-trait association group on 6D was consistent across several data sets, and one marker-trait association group on chromosome 6D was not previously reported. Of the highly resistant landrace accessions, six have novel resistance haplotype profiles. These resistant accessions and haplotype regions can be used to confirm resistance loci in biparental mapping populations for introgression into advanced wheat breeding lines.

Acknowledgements The authors would like to thank Mary Osenga at the Small Grains Genotyping Laboratory in Fargo, ND, for running the $90 \mathrm{~K}$ SNP genotyping on this panel, Shannon Ristrem for her assistance rating the DB nurseries, and Kathy Klos for her thorough review of this manuscript.

Author Contribution statement TC, RW, and JMB and JC were involved in planning, data collection, and analysis. DH coordinated the USU DB trials. HB curated GRIN data and provided germplasm. All authors contributed to the development of the study and preparation of the manuscript.

\section{Compliance with ethical standards}

Conflict of interest On behalf of all authors, the corresponding author states that there is no conflict of interest.
Open Access This article is licensed under a Creative Commons Attribution 4.0 International License, which permits use, sharing, adaptation, distribution and reproduction in any medium or format, as long as you give appropriate credit to the original author(s) and the source, provide a link to the Creative Commons licence, and indicate if changes were made. The images or other third party material in this article are included in the article's Creative Commons licence, unless indicated otherwise in a credit line to the material. If material is not included in the article's Creative Commons licence and your intended use is not permitted by statutory regulation or exceeds the permitted use, you will need to obtain permission directly from the copyright holder. To view a copy of this licence, visit http://creativecommons.org/licenses/by/4.0/.

\section{References}

Admassu-Yimer B, Gordon T, Bonman JM, Klos KE (2019) Development and validation of a quantitative PCR assay method of assessing relative resistance of oat (Avena sativa) to crown rust (Puccinia coronata f.sp. avenae). Plant Pathol 68:669-677

Alaux M, Rogers J, Letellier T, Flores R, Alfama F, Pommier C, Mohellibi N, Durand S, Kimmel E, Michotey C et al (2018) Linking the International Wheat Genome Sequencing Consortium bread wheat reference genome sequence to wheat genetic and phenomic data. Genome Biol 19:111

Babiker EM, Gordon TC, Chao S, Newcomb M, Rouse MN, Jin Y, Waynera R, Acevedo M, Brown-Guedira G, Williamson S, Bonman JM (2015) Mapping resistance to the Ug99 race group of the stem rust pathogen in a spring wheat landrace. Theor Appl Genet 128:605-612

Benjamini Y, Hochberg Y (1995) Controlling the false discovery rate: a practical and powerful approach to multiple testing. J R Stat Soc B 57:289-300

Bhatta M, Morgounov A, Belamkar V, Yorgancilar A, Baenziger PS (2018) Genome-wide association study reveals favorable alleles associated with common bunt resistance in synthetic hexaploid wheat. Euphytica 214:200-210

Bonman JM, Bockelman HE, Goates BJ, Obert DE, McGuire PE, Qualset CO, Hijmans RJ (2006) Geographic distribution of common and dwarf bunt resistance in landraces of Triticum aestivum subsp. aestivum. Crop Sci 46:1622-1629

Burnham KP, Anderson DR (2004) Multimodel inference: understanding AIC and BIC in model selection. Sociol Methods Res 33:261-304

Castlebury LA, Carris LM, Vánky K (2005) Phylogenetic analysis of Tilletia and allied genera in order Tilletiales (Ustilaginomycetes; Exobasidiomycetidae) based on large subunit nuclear rDNA sequences. Mycologia 94:888-900

Chen J, Guttieri MJ, Zhang J, Hole D, Souza E, Goates BJ (2016) A novel QTL associated with dwarf bunt resistance in Idaho 444 winter wheat. Theor Appl Genet 129:2313-2322

Earl DA, vonHoldt BM (2012) STRUCTURE HARVESTER: a website and program for visualizing STRUCTURE output and implementing the Evanno method. Conserv Genet Resour 4:359-361

Evanno G, Regnaut S, Goudet J (2005) Detecting the number of clusters of individuals using the software STRUCTURE: a simulation study. Mol Ecol 14:2611-2620

FAOSTAT (2019) Production and yield quantities of cereal grains. Food and agriculture organization of the United Nations

Gao L, Turner MK, Chao S, Kolmer J, Anderson JA (2016) Genome wide association study of seedling and adult plant leaf rust resistance in elite spring wheat breeding lines. PLoS ONE 11:e0148671 
Goates BJ (1996) Common Bunt and Dwarf Bunt. In: Wilcoxson RD, Saari EE (eds) Bunt and smut diseases of wheat: concepts and methods of disease management. CIMMYT, D.F., pp 12-25

Goates BJ (2012) Identification of new pathogenic races of common bunt and dwarf bunt fungi, and evaluation of known races using an expanded set of differential wheat lines. Plant Dis 96:361-369

Hanson CH, Robinson HF, Cornstock RE (1956) Biometrical studies of yield in segregating populations of Korean Lespedeza. Agron J 48:268-272

Henderson CR (1975) Best linear unbiased estimation and prediction under a selection model. Biometrics 31:423-447

Hiebert CW, Kassa MT, McCartney CA, You FM, Rouse MN, Fobert P, Fetch TG (2016) Genetics and mapping of seedling resistance to Ug99 stem rust in winter wheat cultivar Triumph 64 and differentiation of SrTmp, SrCad, and Sr42. Theor Appl Genet 129:2171-2177

Hoffman JA, Metzger RJ (1976) Current status of virulence genes and pathogenic races of the wheat bunt fungi in the northwestern USA. Phytopathology 66:657-660

Hole DJ, Clawson SM, Young SA, Roche D (2002) Registration of 'Golden Spike' wheat. Crop Sci 42:1376

IWGSC (2018) Shifting the limits in wheat research and breeding using a fully annotated reference genome. Science 361

Kassa M, You FM, Fetch TG, Fobert P, Sharpe A, Pozniak CJ, Menzies JG, Jordan MC, Humphreys G, Zhu T, Lou M-C, McCartney CA, Hiebert CW (2016) Genetic mapping of SrCad and SNP marker development for marker-assisted selection of Ug99 stem rust resistance in wheat. Theor Appl Genet 129:1373-1382

Keener TK, Stougaard RN, Mathre DE (1995) Effect of winter wheat cultivar and difenoconazole seed treatment on dwarf bunt. Plant Dis 79:601-604

Kollmorgen JF, Ballinger J (1987) Detection and morphology of hyphae of common bunt fungi (Tilletia laevis and T. tritici) in wheat seedlings. Trans Br Mycol Soc 88:555-558

Kühn JG (1874) Tilletia contraversa Hedwigia 13:188-189

Liu W, Maccaferri M, Bulli P, Rynearson S, Tuberosa R, Chen X, Pumphrey M (2017) Genome-wide association mapping for seedling and field resistance to Puccinia striiformis f. sp. tritici in elite durum wheat. Theor Appl Genet 130:649-667

Maccaferri M, Zhang J, Bulli P, Abate Z, Chao S, Cantu D, Bossolini E, Chen X, Pumphrey M, Dubcovsky J (2015) A genome-wide association study of resistance to stripe rust (Puccinia striiformis f. sp. tritici) in a worldwide collection of hexaploid spring wheat (Triticum aestivum L.). G3: Genes Genom Genet 114:014563

Menzies JG, Knox RE, Popovic Z, Procunier JD (2006) Common bunt resistance gene Bt10 located on wheat chromosome 6D. Can J Plant Sci 86:1409-1412

Mourad AMI, Sallam A, Belamkar V, Mahdy E, Bakheit B, El-Wafaa AA, Baenziger PS (2018) Genetic architecture of common bunt resistance in winter wheat using genome-wide association study. BMC Plant Biol 18:280-294

Price AL, Patterson RM, Plenge RM, Weinblatt ME, Shadick NA, Reich D (2006) Principal components analysis corrects for stratification in genome-wide association studies. Nat Genet 38:904-909

Pritchard JK, Stephens M, Donnelly P (2000) Inference of population structure using multilocus genotype data. Genetics 155:945-959

Shapiro SS, Wilk MB (1965) An analysis of variance test for normality. Biometrika 52:591-611

Singh RP, Hodson DP, Huerta-Espino J, Jin Y, Bhavani S, Njau P, Herrera-Foessel S, Singh PK, Singh S, Govindan V (2011) The emergence of $\mathrm{Ug} 99$ races of stem rust fungus is a threat to world wheat production. Annu Rev Phytopathol 49:465-491

Singh A, Knox RE, DePauw RM, Singh AK, Cuthbert RD, Kumar S, Campbell HL (2016) Genetic mapping of common bunt resistance and plant height QTL in wheat. Theor Appl Genet 129:243-256

Steffan PM, Torp AM, Borgen A, Backes G, Rasmussen SK (2017) Mapping of common bunt resistance gene $B t 9$ in wheat. Theor Appl Genet 130:1031-1040

Sunderman DW, Hoffman JA, O'Connell BT (1986) Registration of four winter wheat germplasm lines with resistance to dwarf bunt. Crop Sci 26:651-652

Wang SD, Wong D, Forrest K, Allen A, Chao S, Huang E, Maccaferri M, Salvi S, Milner S, Cattievelli L, Mastrangelo AM, Whan A, Stephen S, Barker G, Wieseke R, Plieske J, Consortium IWGS, Lillemo M, Mather D, Apples R, Dolferus R, Brown-Guedira G, Korol A, Akhunova AR, Feuillet C, Salse J, Morgante M, Pozniak C, Ming-Cheng L, Dvorak J, Morell M, Dubcovsky J, Ganal M, Tuberosa R, Lawley C, Mikoulitch I, Cavanagh C, Edwards KE, Hayden M, Akhunov E (2014) Characterization of polyploid wheat genomic diversity using a high-density 90000 single nucleotide polymorphism array. Plant Biotechnol J 12:787-796

Wang R, Gordon T, Hole D, Zhao W, Isham K, Bonman JM, Goates BJ, Chen J (2019) Identification and assessment of two major QTL for dwarf bunt resistance in winter wheat line 'IDO835'. Theor Appl Genet 132:2755-2766

Ward JH Jr, Hook ME (1963) Application of an hierarchical grouping procedure to a problem of grouping profiles. Edu Psychol Meas 23:69-81

Yu J, Pressoir G, Briggs WH, Vroh Bi I, Yamasaki M, Doebley JF, McMullen MD, Gaut BS, Nielsen DM, Holland JB, Kresovich S, Buckler ES (2006) A unified mixed-model method for association mapping that accounts for multiple levels of relatedness. Nat Genet 38:203-208

Zadoks JC, Chang TT, Konzak CF (1974) A decimal code for the growth stages of cereals. Weed Res 14:415-421

Zegeye H, Rasheed A, Makdis F, Badebo A, Ogbonnaya FC (2014) Genome-wide association mapping for seedling and adult plant resistance to stripe rust in synthetic hexaploid wheat. PLoS ONE 9:e105593

Publisher's Note Springer Nature remains neutral with regard to jurisdictional claims in published maps and institutional affiliations. 